\title{
About mathematical models of system dynamics with geometric constraints in problems of stability and stabilization by incomplete state information
}

\begin{abstract}
This article is devoted to constructing equations of motion for Holonomic systems using Lagrangian variables. We introduce the form of equations which allow for detailed analyses of both linear and non-linear terms of perturbed motion equations. Steady motion stability of systems with redundant coordinates is only possible in critical cases; e.g. when the characteristic equation has roots whose real parts are zero. In this case, it is imperative to analyze the nonlinear terms of the characteristic equation to solve stability issues. We suggest a rigorous method of solving stability problem for systems with geometric constraints. The method is based on analytical mechanics, theory of critical cases, nonlinear stability theory, and N.N. Krasovsky's procedure of solving linear-quadratic problems. This grants the ability to make reasonable conclusions regarding system stability and calculations and calculate coefficients of the linear stabilizing control.
\end{abstract}

Volume 2 Issue I - 2017

\section{Krasinskiy Alexandr Ya, Esfira M Krasinkaya, Anastasiya N llyina}

Moscow Aviation Institute, Moscow State technical university BMSTU, Russia

\author{
Correspondence: Krasinskiy Alexandr Ya, Moscow Aviation \\ Institute, Moscow State Food University Moscow, Russia, \\ Email krasinsk@mail.ru
}

Received:September 12,2016 | Published: February 06, 2017

Keywords: geometric constraints, homonymic systems, lagrangian variables, mechatronic systems, routh variables, shulgins equations

\section{Introduction}

Currently, mainstream classes of technical devices consist of controlled mechanical systems with microcontrollers in the control loop. It is possible for such a system to implement, in real time, control algorithms of almost any complexity. The considerations of control problems for modern technical facilities are comprised of three main steps. Firstly, the program control must be defined. This de-termines an object's mode of operation (the dynamics of concrete actuating devices should be taken into account in the framework of the adopted model). Secondly, as perturbations inevitably exist, control is required to stabilize the system. The control can be constructed as a linear function of the state variables. However, the physical state of the system is not wholly determinable by direct observation; it is often technically impossible or inefficient, as each applied sensor raises the cost of the system. Therefore, thirdly, it is required to construct a system that provides an estimate of the system's internal state. This is one of the most important stages to solve many problems in control theory and provides an essential foundation for many practical applications. Suitable and accurate nonlinear mathematical models have fundamental importance at every stage of scrutiny regarding modern technical problems, especially concerning stability and stabilization problems for systems lacking complete state information. Such models are the only reliable basis for the development of rigorous, effective, and more simplistic methods for practical applications. For example, it allows for the possibility of using the characteristics of object's proper motion (without application of additional actions) to reduce the amount of operating actuators and measurement information.

Analytical mechanics are one of the most formalized and developed areas of applied mathematics, and as such, it provides numerous alternatives for the prevailing choices of modeling methods. ${ }^{1-3}$ Generalized coordinates are traditionally used in the study of mechanical systems dynamics. These generalized coordinates are the parameters (in a minimum number) that uniquely define the system's configuration. While there are many choices for system coordinates, it is possible that a useful set of coordinates may be wholly or partially dependent, which means that they are linked by one or more constraint equations. In this case, we cannot directly use Lagrange's equations of motion, as it requires all available coordinates to be independent. This is a commonly encountered problem for multilink manipulators and many other Mechatronic systems in the presence of geometric constraints. ${ }^{4,5}$

It is efficient to describe the configuration of a Mechatronic system with $\mathrm{m}$ geometric constraints by utilizing $\mathrm{n}+\mathrm{m}$ pa-rameters taken in the number exceeding the minimum required number $\mathrm{n}$. These $\mathrm{m}$ parameters are then called redundant coordinates. Detailed analyses of different forms of equations of motion were performed ${ }^{5}$ for systems with geometric constraints. It was shown that the application of equations of motion in M.F. Shul'gin's form ${ }^{6}$ can, quite simply, give rigorous nonlinear mathematical models for systems with redundant coordinates. However, the simplicity of the model is not merely dependent on the chosen coordinates and the form of applied equations of motion.

The type of available variables (Lagrange, Hamilton, or Routh's) ${ }^{1}$ are also critically important. The application of Routh's variables simplifies ${ }^{7-9}$ the determination of stabilizing control coefficients, yet remain very disadvantage ${ }^{8}$ in the case of asymptotic stabilization by all variables in case of incomplete state information. Knowing the perturbations of momenta corresponding to cyclic coordinates is imperative for constructing the control in the case Routh's variables, but they cannot be determined by direct measurements. Lagrangian variables are useful in terms of reducing the amount of measuring information as generalized velocities can be determined directly by measuring devices. But the application of Lagrangian variables substantially complicates the determination of stabilizing control coefficients and makes it difficult to undertake an analysis 
of equations. In this case, the number of characteristic equation zero roots is more than the number of geometric constraints. Thus, the structure of nonlinear closed loop system (in the special form of critical cases theory ${ }^{10,11}$ ) is clear in Routh's variables but is difficult for analyses in Lagrangian variables. As the first step (determination of the stabilizing control coefficients in Routh's variables) was studied in detail, ${ }^{8,9,12}$ the main idea of this article is constructing the nonlinear model of systems dynamics in Lagrangian variables in the presence of cyclic coordinates. Then a theorem on solvability of the stabilization problem for stationary motion is proved in case of incomplete state information.

\section{MF shulgins equations}

Suppose configuration of a mechanical system is described by parameter $q^{1} ; ; ; ; ; q^{n+m}$ where $\mathrm{n}$ is the number of the system degrees of freedom. Let the system configuration is limited by $\mathrm{m}$ independent relations (geometric constraints)

$$
\begin{aligned}
& \mathrm{F}_{k}\left(q_{1}, \ldots q_{n+m}\right)=0 ; \quad \mathrm{k}=\overline{1, m} \\
& \operatorname{det} \frac{\partial\left[F_{1}, . . F_{m}\right]}{\partial\left[q_{n+1}, \ldots, . q_{n+m}\right]} \neq 0
\end{aligned}
$$

Without loss of generality coordinates $q^{n+1} \ldots \ldots q^{n+m}$ can be considered as redundant. Introduce some vectors (the prime denotes transposition):

$$
\begin{aligned}
& r^{\prime}=\left(q_{1}, \ldots, q_{n}\right), s^{\prime}=\left(q_{n+1}, \ldots, q_{n+m}\right), q^{\prime}=\left(r^{\prime}, s^{\prime}\right), \\
& F^{\prime}=\left(F_{1}, \ldots, F_{m}\right) ;
\end{aligned}
$$

Suppose the system is affected by potential forces with energy and no potential forces $Q \sim r ; Q \sim s$ corresponded to coordinates; s and $\sim L=\sim$ T $\square$ is Lagrange function. The general view

Of the kinetic energy is:

$$
\begin{aligned}
& \tilde{\mathrm{T}}=\tilde{\mathrm{T}}_{2}+\tilde{\mathrm{T}}_{1}+\tilde{\mathrm{T}}_{0}=\frac{1}{2} \dot{q}^{\prime} \tilde{\mathrm{a}}(\mathrm{q}) \dot{\mathrm{q}}+\tilde{\mathrm{d}}^{\prime}(\mathrm{q}) \dot{\mathrm{q}}+\mathrm{T}_{0}(q) \\
& \tilde{\mathrm{a}}(\mathrm{q})=\left(\begin{array}{ll}
\tilde{\mathrm{a}}_{\mathrm{rr}} & \tilde{\mathrm{a}}_{\mathrm{rs}} \\
\tilde{\mathrm{a}}_{\mathrm{s} r} & \tilde{\mathrm{a}}_{\mathrm{ss}}
\end{array}\right) ; \quad \tilde{\mathrm{d}}(\mathrm{q})=\left(\begin{array}{c}
\tilde{\mathrm{d}}_{\mathrm{r}} \\
\tilde{\mathrm{d}}_{\mathrm{s}}
\end{array}\right)
\end{aligned}
$$

Kinematic (Holonomic) constraints can be obtained by differentiating the geometric constraint equations (1) with respect to time:

$$
\frac{\partial F}{\partial r^{\prime}} \dot{r}+\frac{\partial F}{\partial s^{\prime}} \dot{s}=0
$$

The depended velocities vector can be expressed from (3):

$$
\dot{s}=B(q) \dot{r} ; B(q)=-\left(\frac{\partial F}{\partial s^{1}}\right)^{-1} \cdot\left(\frac{\partial F}{\partial r^{1}}\right)
$$

Let or $(q \ddot{y} r), Q s(q \ddot{y} r) \quad L(q \ddot{y} r)=T(q \ddot{y} r)-\Pi$ denote the no potential forces and Lagrange function after eliminating the dependent velocities using (4).

$$
T(q, \dot{r})=T_{2}+T_{1}+T_{0}=\frac{1}{2} \dot{r}^{\prime} a(q) \dot{r}+d^{\prime}(q) \dot{r}+T_{0}(q),
$$

$$
a(q)=\tilde{a}_{r r}+2 \cdot \tilde{a}_{r s} B+B \tilde{a}_{s s} B, d^{\prime}(q)=\tilde{d}_{r}^{\prime}+\tilde{d}_{s}^{\prime} B .
$$

Then the equations in M.F. Shul'gin's from [5], [6] in Lagrangian variables can be written as

$$
\begin{gathered}
\frac{d}{d t} \frac{\partial L}{\partial \dot{r}}-\frac{\partial L}{\partial r}=Q_{r+} B^{\prime}(q)\left(\frac{\partial L}{\partial s}+Q_{s}\right) ; \\
\dot{s}=B(q) \dot{r}
\end{gathered}
$$

\section{M.F. shul'gin's equations for systems with cyclic coordinates}

Suppose the coordinates_are cyclic in terms ${ }^{6}$

$$
\begin{aligned}
& \frac{\partial F}{\partial \beta}=0, \frac{\partial B}{\partial \beta}=0, \frac{\partial T}{\partial \beta}=0, \frac{\partial \Pi}{\partial \beta}=0, Q_{\beta}=0 . \\
& \dot{s}=-\left(\frac{\partial F}{\partial s^{\prime}}\right)^{-1}\left(\frac{\partial F}{\partial \alpha^{\prime}}\right) \dot{\alpha}=B_{\alpha}(\alpha, s) \dot{\alpha} .
\end{aligned}
$$

As cyclic velocities are not included in the equations of the constraints, using (7), we obtain the kinetic energy (2) in the form

$$
\begin{aligned}
& \breve{T}(\alpha, s . \dot{\alpha}, \beta)=\frac{1}{2}\left(\begin{array}{ll}
\dot{\alpha}^{\prime} & \dot{\beta}^{\prime}
\end{array}\right)\left(\begin{array}{ll}
\vec{a}_{\alpha \alpha} & \dot{a}_{\alpha \beta} \\
\vec{a}_{\beta \alpha} & \vec{a}_{\beta \beta}
\end{array}\right)\left(\begin{array}{c}
\dot{\alpha} \\
\dot{\beta}
\end{array}\right)+\left(\begin{array}{ll}
\breve{d}_{\alpha}^{\prime} & \breve{d}_{\beta}^{\prime}
\end{array}\right)+T_{0} \\
& \tilde{a}(\alpha, s)=\left(\begin{array}{ccc}
\tilde{a}_{\alpha \alpha} & \tilde{a}_{\alpha \beta} & \tilde{a}_{\alpha \beta} \\
\tilde{a}_{\beta \alpha} & \tilde{a}_{\beta \beta} & \tilde{a}_{\beta s} \\
\tilde{a}_{s \alpha} & \tilde{a}_{s \beta} & \tilde{a}_{s s}
\end{array}\right), \tilde{d}=\left(\begin{array}{c}
\tilde{d}_{\alpha} \\
\tilde{d}_{\beta} \\
\tilde{d}_{s}
\end{array}\right) \\
& \tilde{a}_{\alpha \alpha}=\tilde{a}_{\alpha \alpha}+2 \tilde{a}_{\alpha s} B_{\alpha}+B_{\alpha}^{\prime} a_{s s} B_{\alpha} ; \breve{a}_{\alpha \beta}=\tilde{a}_{\alpha \beta}+B_{\alpha}^{\prime} \tilde{a}_{s \beta} ; \\
& \breve{a}_{\beta \alpha}=\breve{a}_{\alpha \beta} ; \breve{a}_{\beta \beta}=\breve{a}_{\beta \beta} ; \breve{d}_{\alpha}=\tilde{d}_{s} B ; \breve{d}_{\beta}^{\prime}=\tilde{d}_{\beta}^{\prime}
\end{aligned}
$$

Lagrange's function is $\quad i L(\alpha, s, \ddot{\mathrm{y}} \alpha, \ddot{\mathrm{y}} \beta)=i T-\Pi$. Then theequations (6) can be written as

$$
\begin{gathered}
\frac{d}{d t} \frac{\partial \breve{L}}{\partial \dot{\alpha}}-\frac{\partial \breve{L}}{\partial \alpha}=Q_{\alpha}+B_{\alpha}^{\prime}\left(\frac{\partial \breve{L}}{\partial s}+Q_{s}\right) ; \\
\frac{d}{d t} \frac{\partial \breve{L}}{\partial \dot{\beta}}=0 \\
\dot{s}=B_{\alpha}(\alpha, \mathrm{s}) \dot{\alpha} .
\end{gathered}
$$

As obviously follows from (8) the system has cyclic integrals and holonomic systems always ${ }^{1,2,6}$ have steady motions

$$
\begin{aligned}
& \dot{\alpha}=0 ; \quad \alpha=\alpha_{0}=\text { const } ; \\
& s=s_{0}=\text { const } ; \quad \dot{\beta}=c_{\beta}=\text { const } .
\end{aligned}
$$




\section{Equations of perturbed motion in the neighborhood of a steady motion}

Introduce some initial perturbations:

$$
\alpha=\alpha_{0}+x ; s=s_{0}+y ; \dot{\beta}=c_{\beta}+\omega
$$

And assume that no potential forces corresponding to the position coordinates have the following structure:

$$
\begin{aligned}
& \tilde{Q}_{\alpha}^{*}=f_{\alpha \alpha} \dot{\alpha}+f_{\alpha s} \dot{s}+g_{\alpha \alpha} \alpha+g_{\alpha s} s+\tilde{Q}_{\alpha}^{(2)} ; \\
& \tilde{Q}_{s}^{*}=f_{s \alpha} \dot{\alpha}+f_{s s} \dot{s}+g_{s \alpha} \alpha+g_{s s} s+\tilde{Q}_{s}^{(2)} .
\end{aligned}
$$

Then, the vector-matrix equations perturbed motion with separated first approximation can be written as

$$
\begin{aligned}
& A_{1} \ddot{\chi}+A_{2} \dot{\omega}+ \\
& +\left(c_{\beta}^{\prime} \psi_{1}+D_{\alpha}-F_{\alpha}\right) \dot{\chi}+\left(c_{\beta}^{\prime} \psi_{2}+D_{\beta}-F_{\beta}\right) \omega+ \\
& +\left[C_{1}-G_{s}+\left(\frac{\partial B_{\mu \chi}}{\partial q_{\chi}}\right)_{0}\left(\frac{\partial W}{\partial q_{\mu}}\right)_{0}+B_{\beta}^{\prime}(0) C_{2}+c_{\beta}^{\prime} \varphi_{2}^{1} c \beta-G_{\alpha}\right] \chi+ \\
& +\left[C_{3}\left(\frac{\partial B_{\kappa \chi}}{\partial q_{\mu}}\right)_{0}\left(\frac{\partial W}{\partial q_{\kappa}}\right)_{0}+B_{\alpha}^{\prime}(0) C_{4}+c_{\beta}^{\prime} \varphi_{2}^{2} c_{\beta}\right] y=X_{\alpha}^{(2)}(x, y, \dot{x}, \omega) \\
& \dot{y}=B_{\alpha}(0) \dot{\chi}+B_{\alpha}^{(1)}(\chi, y) \dot{\chi} ; \\
& A_{3} \ddot{x}+A_{4} \dot{\omega}+\left(c_{\beta}^{\prime} \psi_{3}+\tilde{D}_{\beta}\right) \dot{x}=X_{\beta}^{(2)}(x, y, \dot{x}, \omega) ; \\
& B_{\alpha}^{(1)}(x, y)=B_{\alpha}\left(\alpha_{0}+x, s_{0}+y\right)-B_{\alpha}(0) ; \\
& A_{1}=\left\|a_{\gamma \delta}(0)\right\|, A_{2}=\left\|a_{\gamma \sigma}(0)\right\|, A_{3}=\left\|a_{\sigma \delta}(0)\right\|, \\
& A_{4}=\left\|a_{\sigma \gamma}(0)\right\| ; \quad C_{1}=\left(\frac{\partial^{2} W}{\partial q_{x} \partial q_{\gamma}}\right)_{0} \\
& C_{2}=\left(\frac{\partial^{2} W}{\partial q_{x} \partial q_{\mu}}\right)_{0}, C_{4}=\left(\frac{\partial^{2} W}{\partial q_{\mu} \partial q_{\kappa}}\right)_{0}, C_{3}=\mathrm{C}_{2}^{\prime} ; \\
& D_{\alpha}=\left\|\frac{\partial d_{\gamma}}{\partial q_{x}}+\frac{\partial d_{\gamma}}{\partial q_{\mu}} B_{\mu x}-\frac{\partial d_{x}}{\partial q_{x}}-\mathrm{B}_{\mu \gamma} \frac{\partial d_{x}}{\partial q_{\gamma}}\right\| 0 \\
& D_{\beta}=\left\|-\left(\frac{\partial d_{\sigma}}{\partial q_{\gamma}}+B_{\mu \gamma} \frac{\partial d_{\sigma}}{\partial q_{\mu}}\right)\right\| 0 \text {, }
\end{aligned}
$$

$$
\begin{aligned}
& F_{\alpha}=f_{\alpha \alpha}+f_{\alpha s} B_{\alpha}(0)\left(f_{s \alpha}+f_{s s} B_{\alpha}(0)\right), \\
& \mathrm{F}_{\beta}=f_{\alpha \beta}+B_{\alpha}^{\prime}(0) f_{s \beta} ; \quad W=\Pi-T_{0} ; \\
& G_{\alpha}=g_{\alpha \alpha}+B_{\alpha}^{\prime}(0) g_{s \alpha}, \quad G_{s}=g_{\alpha s}+B_{\alpha}^{\prime}(0) g_{s s}, \\
& \psi_{1}=\left\|\frac{\partial a_{\gamma \alpha}}{\partial q_{x}}+\frac{\partial a_{\gamma \alpha}}{\partial q_{\mu}} B_{\mu \chi}-\frac{\partial a_{\chi \alpha}}{\partial q_{\gamma}}-B_{\mu} \frac{\partial a_{\chi \sigma}}{\partial q_{\mu}}\right\| 0, \\
& \psi_{2}=\left\|\psi_{\gamma \sigma}(0)\right\|, \psi_{\gamma \sigma}=-\left(\frac{\partial a_{\gamma \alpha}}{\partial q_{\gamma}} B_{\mu \gamma} \frac{\partial a_{\chi \alpha}}{\partial q_{\mu}}\right), \\
& \psi_{2}^{1}=\frac{1}{2}\left\|\frac{\partial \psi_{\gamma \sigma}}{\partial q_{\mu}}\right\| 0_{3}, \quad \psi_{2}^{2}=\frac{1}{2}\left\|\frac{\partial \psi_{\gamma \sigma}}{\partial q_{\mu}}\right\| 0 \\
& \psi_{3}=\left\|\frac{\partial a_{\gamma \sigma}}{\partial q_{\chi}}+\frac{\partial a_{\gamma \sigma}}{\partial q_{\mu}} B_{\mu \chi}\right\|_{0}, \quad \tilde{D}{ }_{\beta}=\left\|\frac{\partial d_{\chi}}{\partial q_{\chi}}+\frac{\partial d_{\chi}}{\partial q_{\mu}} B_{\mu x}\right\|_{0},
\end{aligned}
$$

Repeated indexes are summed over the values:

$$
\begin{aligned}
& \gamma, \delta, \chi=1, \ldots, l ; \xi, \alpha, \mathrm{r}=l+1, \ldots, n \\
& \mu, \mathrm{k}=n+1, \ldots, n+m .
\end{aligned}
$$

Determine variables corresponding to zero roots of the characteristic equation via the linear substitution [5]:

$$
z=y-B_{\alpha}(0) x .
$$

The system (10) can be written as

$$
A_{1} \ddot{x}+A_{2} \dot{\omega}+\left(\Phi_{\alpha}-F_{\alpha}\right) \dot{x}+\left(\Gamma-F_{\beta}\right) \omega+\mathrm{K} x+\mathrm{Sz}=\mathrm{X}^{(2)}(x, z, \dot{x}, \omega)
$$

$$
\begin{gathered}
A_{3} \ddot{x}+A_{4} \dot{\omega}+\Phi_{\beta} \dot{x}, \quad \dot{z}=\mathrm{B}_{\alpha}^{(1)}(x, z) \dot{x} ; \\
\Phi_{\alpha}=c_{\beta}^{\prime} \Psi_{1}+D_{\alpha}, \quad \Phi_{\beta}=c_{\beta}^{\prime} \Psi_{3}+\tilde{D}_{\beta}, \\
\Gamma=c_{\beta}^{\prime} \Psi_{2}+D_{\beta} ;
\end{gathered}
$$$$
\mathrm{K}=C_{1}+\mathrm{B}_{\alpha}^{\prime}(0) C_{2}+C_{3} \mathrm{~B}_{\alpha}(0)+\mathrm{B}_{\alpha}^{\prime}(0) C_{4} \mathrm{~B}_{\alpha}(0)+
$$$$
+C^{\mathrm{B}}+c_{\beta}^{\prime} \Psi_{2}^{1} c_{\beta}+c_{\beta}^{\prime} \Psi_{2}^{2} c_{\beta} \mathrm{B}(0)-G_{\alpha}-G_{s} \mathrm{~B}_{\alpha}(0) ;
$$ 


$$
C^{\mathrm{B}}=\left(\frac{\partial \mathrm{B}_{\mu \gamma}}{\partial q_{x}}\right)_{0}\left(\frac{\partial W}{\partial q_{\mu}}\right)_{0}+\left(\frac{\partial \mathrm{B}_{\kappa \gamma}}{\partial q_{\mu}}\right)_{0}\left(\frac{\partial W}{\partial q_{\kappa}}\right)_{0} \mathrm{~B}_{\alpha}(0)
$$

It is necessary to pay attention on the matrix $\mathrm{CB}$. Its components depend on second-order derivatives of the geometric constraint equations (1).

\section{Stabilization of a steady motion for systems with incomplete state information}

Consider the case when the control action $\mathrm{u}$ acts on by all components of cyclic coordinate's vector. The normal form of the perturbed motion equations is:

$$
\begin{aligned}
& \dot{\xi}=\mathrm{N} \xi+\mathrm{Vu}+\mathrm{Zz}+\Xi^{(2)}(\xi, z), \dot{z}=\mathrm{B}_{\alpha}^{(1)}(x, z) x_{1} \\
& \xi^{\prime}\left(x^{\prime}, x_{1}^{\prime}, \omega^{\prime}\right), \quad \mathrm{N}=\left(\begin{array}{cc}
\mathrm{I} & 0 \\
-\mathrm{A}^{-1} \tilde{\mathrm{K}} & -\mathrm{A}^{-1} \mathrm{P}
\end{array}\right) ; \\
& \mathrm{P}=\left(\begin{array}{cc}
\Phi_{\alpha}-F_{\alpha} & \Gamma-F_{\beta} \\
\Phi_{\beta} & 0
\end{array}\right), \quad \tilde{\mathrm{K}}=\left(\begin{array}{c}
\mathrm{K} \\
0
\end{array}\right), \\
& V=\left(\begin{array}{c}
0 \\
\mathrm{~A}^{-1} \mathrm{M}
\end{array}\right), \mathrm{M}=\left(\begin{array}{c}
0 \\
\mathrm{E}_{\eta-1}
\end{array}\right), \mathrm{A}=\left(\begin{array}{ll}
\mathrm{A}_{1} & \mathrm{~A}_{2} \\
\mathrm{~A}_{3} & \mathrm{~A}_{4}
\end{array}\right) \\
& \mathrm{Z}=\left(\begin{array}{c}
0 \\
\mathrm{~A}^{-1} \tilde{S}
\end{array}\right), \mathrm{I}=\left(\begin{array}{ll}
0 & \mathrm{E}_{\imath}
\end{array}\right), \quad \tilde{S}=\left(\begin{array}{l}
S \\
0
\end{array}\right), \\
& \Xi^{(2)}=\left(\begin{array}{c}
0 \\
A^{-1} \tilde{\Xi}^{(2)}
\end{array}\right), \quad \tilde{\Xi}^{(2)}=\left(\begin{array}{c}
X^{(2)} \\
0
\end{array}\right) \text {. }
\end{aligned}
$$

The simplest variants of matrices of coefficients for linear approximation of measurement vector are $\sigma i=\Sigma i \xi$ :

$$
\Sigma_{1}=\left(\begin{array}{lll}
\mathrm{E}_{\imath} & 0 & 0
\end{array}\right) ; \quad \Sigma_{2}=\left(\begin{array}{lll}
0 & \mathrm{E}_{\imath} & 0
\end{array}\right) ; \Sigma_{3}=\left(\begin{array}{lll}
0 & 0 & \mathrm{E}_{\eta-l}
\end{array}\right) ;
$$

The observing system is:

$$
\dot{\hat{\xi}}=\mathrm{N} \hat{\xi}+\mathrm{Vu}-\mathrm{L}_{\kappa}\left(\Sigma_{\kappa} \hat{\xi}-\sigma_{\kappa}\right), \tilde{\xi}=\left(\hat{x}^{\prime}, \hat{x}_{1}^{\prime}, \hat{\omega}^{\prime}\right) ;
$$

It provides an estimate of the phase state of system (13). The vector $^{\wedge} \xi k$ is obtained from the measurement $\sigma k$ by the solution of the stabilization dual problem

$$
\dot{\mu}_{\kappa}=\mathrm{N}^{\prime} \mu_{\kappa}+\Sigma_{\kappa}^{\prime} v_{\kappa}, \quad v_{\kappa}=L_{\kappa}^{\prime} \mu_{\kappa} .
$$

Theorem 1: If the following conditions

$$
\begin{gathered}
\operatorname{rank}\left(V \mathrm{~N} V \mathrm{~N}^{2} V \ldots \mathrm{N}^{\eta+t-1} V\right)=\eta+\imath ; \\
\operatorname{rank}\left(\Sigma_{\kappa}^{\prime} \mathrm{N}^{\prime} \Sigma_{\kappa}^{\prime} \mathrm{N}^{2} V \ldots \mathrm{N}^{\eta+t-1} \Sigma_{\kappa}^{\prime}\right)=\eta+\imath ; \kappa=1,2,3 ;
\end{gathered}
$$

Are met for system (17) then there is a linear control

$$
u_{\kappa}=\Lambda_{\kappa} \hat{\xi}_{\kappa}
$$

Stabilizing steady motion (13) to asymptotic stability with respect to all variables. It should be noted that in Routh's variables the third column of the matrix of measurements is always consists of zeros. Obviously, any linear combination of columns is possible for matrix of measurements in Lagrangian variables $\Sigma=(a \Sigma 1+b \Sigma 2+c \Sigma 3)$. Therefore, the use of Lagrangian variables can provide further capacity to full the conditions of observability compared with variables Routh's.

\section{Conclusion}

This work develops results. ${ }^{5,8,9,12}$ Mathematical model of a system with geometric constraints was constructed using Shul'gin's equations of motion and Lagrangian variables. Besides, we suggested that some of coordinates are cyclic. The equations were presented in general form. The advantages of this form of equations for solving stability and stabilization problems were discussed. Then the theorem about solvability of the stabilization problem for steady motions was formulated in case of systems with incomplete state information. This result can be applied for solving stability and stabilization problems for many mechanical systems such as multilink manipulators or other systems with geometric constraints.

\section{Acknowledgements}

None.

\section{Conflict of interest}

The author declares no conflict of interest.

\section{References}

1. Rumyancev V V. Stability of stationary motion of satellites. USSR academy of science commutating center, Moscow, Russia: 1967. 141 p.

2. Lur'e AI. Analytical mechanics. Russia: State publishing house of mathematical literature; $1961.824 \mathrm{p}$.

3. Suslov GK. Theoretical mechanics. Leningrad, OGIZ, Russia: 1946. 656 p.

4. Zenkevich SL Jushhenko AS. The basis of manipulators control. Russia: Bauman MSTU Publishing House; 2004. 480 p.

5. Krasinskaya Ye M Krasinskiy A Ya. Science and Education. Bauman MSTU. Russia: Publishing House; 2013. p. 347-376.

6. Shul'gin MF. On some differential equations of analytical dynamics and their integration. Russia: Lenin Central Asian state University iss 144T ashkent SASU Publ; 1958. 183 p.

7. Klokov AS, Samsonov VA. About the stabilization of gyroscopically constrained systems with coordinates of pseudocyclic trivial steady motions. 1985;49(2):199-202.

8. Krasinskiy A Ya. Stabilization of steady motions of systems with cyclic coordinates. Mathematics and Mechanics. 1991;56:939-950.

9. Krasinskii A Ya, Krasinskaya EM. The stability and stabilization of nonisolated steady motions of mechanical systems, Holonomic systems. UlSTU Publ. 2011;77(8):301-322. 
10. Ljapunov AM, Sobranie Sochinenij. Collected Works. USSR Academy of Science Commutating Center. 1956;2:481.

11. Malkin I G. The Theory of Dynamic Stability Science, Russia: Springer; 1966.
12. Krasinskiy A Ya, Krasinskaya Ye. System Dynamics and Control Processes. International Conference dedicated to the 90th Anniversary of Academicain; Ekaterinburg, Russia: IEEE; 2014. p. 228-235. 